\title{
On Caputo modification of Hadamard-type fractional derivative and fractional Taylor series
}

\author{
Rashida Zafar', Mujeeb ur Rehman ${ }^{1}$ and Moniba Shams ${ }^{1 *}$ (D)
}

\section{"Correspondence:}

monibashams@gmail.com

'Department of Mathematics,

School of Natural Sciences, National

University of Sciences and

Technology, Islamabad, Pakistan

\begin{abstract}
In this paper a general framework is presented on some operational properties of Caputo modification of Hadamard-type fractional differential operator along with a new algorithm proposed for approximation of Hadamard-type fractional integral using Haar wavelet method. Moreover, a generalized Taylor expansion based on Caputo-Hadamard-type fractional differential operator is also established, and an example is presented for illustration.
\end{abstract}

Keywords: Fractional order calculus; Fractional operators; Caputo modification; Hadamard-type; Fractional Taylor formula

\section{Introduction}

The history of fractional operators dates back to the seventeenth century [1]. However, interest in this theory gained momentum after the publication of [2]. Later, Riemann constructed an integral-based fractional operator [3] which is the well-known RiemannLiouville ( $\mathrm{R}-\mathrm{L}$ ) integral. It proved to be a valuable research in fractional calculus and is being used extensively in this field ever since. The readers are referred to a comprehensive background history of fractional calculus and details on the R-L operators given in [4].

In the last two decades scientists and researchers presented findings dealing with the fractional calculus theory and found that integer order derivatives do not work adequately in many cases. Fractional calculus has played a very important role in fields such as turbulent fluid flows, blood flow through biological tissues, control theory, and signal and image processing, fractional Brownian motion, power law models, history-dependent process, porous media, fractional filters, etc. The detailed literature including theory and applications of fractional calculus can be found in [5-11].

There are different definitions of fractional derivatives available in the literature. However, the most commonly used is the Hadamard fractional derivative given by Hadamard [12]. Butzer et al. [13-16] studied various properties of Hadamard-type derivatives which are more generalized than the Hadamard fractional derivatives. In this context, the readers are also referred to [1] for a detailed study on generalized fractional derivatives and references therein. Caputo introduced another type of fractional derivative [17] which has

(c) The Author(s) 2020. This article is licensed under a Creative Commons Attribution 4.0 International License, which permits use, sharing, adaptation, distribution and reproduction in any medium or format, as long as you give appropriate credit to the original author(s) and the source, provide a link to the Creative Commons licence, and indicate if changes were made. The images or other third party material in this article are included in the article's Creative Commons licence, unless indicated otherwise in a credit line to the material. If material is not included in the article's Creative Commons licence and your intended use is not permitted by statutory regulation or exceeds the permitted use, you will need to obtain permission directly from the copyright holder. To view a copy of this licence, visit http://creativecommons.org/licenses/by/4.0/. 
an advantage over $\mathrm{R}-\mathrm{L}$ derivative in a differential equation since it does not require to define the fractional order initial conditions (see, for example, [18-21]). Recently the authors in $[22,23]$ utilized Caputo-type modification on Hadamard factional derivatives. Moreover Trujillo et al. in [24] derived Taylor's formula with Riemann-Liouville derivatives, and Odibat and Shawagfeh [25] derived the same based on Caputo fractional derivative. Gulsu et al. [26] extended the work of previous authors and proposed a numerical scheme to approximate solutions of relaxation-oscillation equation by using the fractional Taylor series. Finally, Fernandez and Baleanu [27] developed mean value theorem and Taylor's theorem for certain fractional differential operators.

The present research presents an extension of Haar wavelet method to approximate Hadamard-type fractional integrals for a wide range of functions, and a new operational matrix of fractional integration of Haar wavelets is derived for Hadamard-type fractional integrals. A Caputo modification of Hadamard-type fractional derivative, motivated through [22, 23], is also carried out in this work, and composition properties of Caputo modification of Hadamard-type fractional derivative are discussed in detail. Lastly, motivated by [28] and the aforementioned studies, we also establish a generalized Taylor expansion based on Caputo-Hadamard-type fractional differential operators and present an example for illustration.

\section{Preliminaries}

In this section some definitions and basic concepts needed in the sequel are presented precisely. We refer the interested reader to Samko et al. [3] and Kilbas et al. [7] for more details.

The left-sided Hadamard fractional integral is defined as follows.

Definition 2.1 For $f \in L_{p}[a, b], 1 \leq p<\infty$, the Hadamard fractional integral of order $\alpha>0$ is defined as

$$
\mathfrak{I}_{a}^{\alpha} f(x)=\frac{1}{\Gamma(\alpha)} \int_{a}^{x}\left(\log \left(\frac{x}{t}\right)\right)^{\alpha-1} f(t) \frac{d t}{t}, \quad a>0 .
$$

Hadamard fractional derivative of order $\alpha>0$ is given by

$$
\mathfrak{D}_{a}^{\alpha} f(x)=\delta^{m}\left(\mathfrak{I}_{a}^{m-\alpha} f\right)(x)
$$

such that $m=\lceil\alpha\rceil$ and $\delta=x \frac{d}{d x}$.

Definition 2.2 ([15]) For $\delta^{m} f \in L_{p}[a, b]$, the Caputo-Hadamard fractional derivative of order $\alpha>0$ is defined as

$$
C_{\mathfrak{D}_{a}^{\alpha}} f(x)=\frac{1}{\Gamma(m-\alpha)} \int_{a}^{x}\left(\log \frac{x}{t}\right)^{m-\alpha-1}\left(t \frac{d}{d t}\right)^{m} f(t) \frac{d t}{t}=\left(\mathfrak{I}_{a}^{m-\alpha} \delta^{m} f\right)(x),
$$

where $\delta^{m}=\left(x \frac{d}{d x}\right)^{m}$ and $m=\lceil\alpha\rceil$.

We introduce the notation $\delta=x \frac{d}{d x}$ and $\mathfrak{D}_{c}=x^{-c} \delta$, where $c \in \mathbb{R}$. Define a space of functions

$$
\mathrm{AC}_{c}^{m}[a, b]=\left\{f:[a, b] \rightarrow \mathbb{R} \mid \mathfrak{D}^{m-1} f(x) \in \mathrm{AC}[a, b]\right\}
$$


where $\mathrm{AC}[a, b]$ is the space of absolutely continuous functions. Note that $\mathrm{AC}_{0}^{m}[a, b]=$ $\mathrm{AC}^{m}[a, b]$. Furthermore, for $c \in \mathbb{R}, 1 \leq p<\infty$, define the space [18]

$$
\mathfrak{L}_{c}^{p}[a, b]=\left\{f:[a, b] \rightarrow \mathbb{R} \mid\left(\int_{a}^{b}\left|x^{c} f(x)\right|^{p} \frac{d x}{x}\right)^{p}<\infty\right\} .
$$

Remark 2.3 If $f \in \operatorname{AC}_{c}^{m}[a, b]$, then there exists a function $g \in \mathfrak{L}_{c}^{1}[a, b]$ such that

$$
\mathfrak{D}_{c}^{m-1} f(x)=\mathfrak{D}_{c}^{m-1} f(a)+\int_{a}^{b} g(x) d x
$$

where $g$ is a generalized derivative of $f$, i.e., $g(x)=\mathfrak{D}_{c}^{m} f(x)$.

The Hadamard-type fractional integral was introduced in [15]. This operator is formally defined as follows.

Definition 2.4 For $\alpha>0, c \in \mathbb{R}$, the Hadamard-type fractional integral is defined as

$$
\mathfrak{I}_{a, c}^{\alpha} f(x)=\frac{1}{\Gamma(\alpha)} \int_{a}^{x}\left(\frac{s}{x}\right)^{c}\left(\log \frac{x}{s}\right)^{\alpha-1} f(s) \frac{d s}{s} .
$$

For $c=0$, the integral $\mathfrak{I}_{a, d}^{\alpha} f(x)$ reduces to the standard Hadamard fractional integral $\Im_{a, f}^{\alpha} f(x)$.

Lemma 2.5 Let $f \in \mathfrak{L}_{c}^{1}[a, b]$ and $\alpha>0$. Then integral $\mathfrak{I}_{a, c}^{\alpha} f(x)$ exists and $\mathfrak{I}_{a, b}^{\alpha} f(x) \in \mathfrak{L}_{c}^{1}[a, b]$.

The Hadamard-type fractional differential operator is defined as follows.

Lemma 2.6 For $\beta>0, c \in R$, let $f(x)=x^{-c}\left(\log \frac{x}{a}\right)^{\beta}$. Then

$$
\mathfrak{I}_{a, d}^{\alpha} f(x)=\frac{\Gamma(\beta+1) x^{-c}}{\Gamma(\beta+\alpha+1)}\left(\log \frac{x}{a}\right)^{\alpha+\beta} .
$$

Definition 2.7 Let $f \in \mathfrak{L}_{c}^{1}[a, b]$ and $m-1<\alpha \leq m, m \in \mathbb{N}$. Then the Hadamard-type fractional derivative of $f$ is defined by

$$
\mathfrak{D}_{a, c}^{\alpha} f(x)=\mathfrak{D}_{c}^{m} \mathfrak{I}_{a, c}^{m-\alpha} f(x) .
$$

Definition 2.8 Let $f \in \operatorname{AC}_{c}^{m}[a, b]$ and $m-1<\alpha \leq m, m \in \mathbb{N}$. Then the Caputo modification of Hadamard-type derivative is given by

$$
C_{\mathfrak{D}_{a, c}^{\alpha}} f(x)=\mathfrak{D}_{a, c}^{\alpha}\left[f(x)-a^{c} x^{-c} \sum_{k=0}^{m-1} \frac{\mathfrak{D}_{c}^{k} f(a)}{\Gamma(k+1)}\left(\log \frac{x}{a}\right)^{k}\right] .
$$

Lemma 2.9 For $\alpha \geq 0, m=\lceil\alpha\rceil$, and $\mu>-1$, consider the function

$$
f(x)=x^{-c}\left(\log \frac{x}{a}\right)^{\mu}
$$


the Caputo-Hadamard-type derivative off is given by

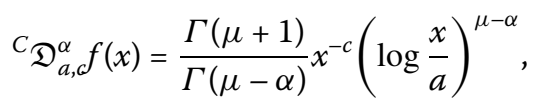

if $\mu \in \mathbb{N}$ and $\mu \geq n$, or $\mu \notin \mathbb{N}$ and $\mu>m-1$. Otherwise

$$
{ }^{C} \mathfrak{D}_{a, c}^{\alpha} f(x)=0,
$$

if $\mu \in\{0,1,2,3, \ldots, m-1\}$.

\section{Approximation of Hadamard-type fractional integral}

Wavelets have been used for numerical integration by several researchers. Based on wavelets, Hashish et al. [29] introduced a method for obtaining approximate value of integrals. In [30, 31], Aziz et al. formulated a numerical integration scheme based on Haar wavelets and hybrid functions. In this section, we develop a method for approximating the Hadamard-type fractional integral. The method presented here is based on operational matrices of fractional integration. The operational matrix of integration for Hadamardtype fractional integral is introduced as follows.

For $x \in[a, b]$, the modified $i$ th Haar wavelet is defined as

$$
\mathcal{H}_{i}(x)= \begin{cases}x^{-c}, & x \in\left[\eta_{1}(i), \eta_{2}(i)\right) ; \\ -x^{-c}, & x \in\left[\eta_{2}(i), \eta_{3}(i)\right) ; \\ 0, & \text { elsewhere }\end{cases}
$$

where $c \in \mathbb{R}$,

$$
\eta_{1}(i)=x(2 k v), \quad \eta_{2}(i)=x((2 k+1) v), \quad \eta_{3}(i)=x((2 k+2) v),
$$

where

$$
\begin{array}{ll}
v=\frac{M}{m}, & k=0,1,2, \ldots, m-1, \quad m=2^{j}, \quad j=0,1,2, \ldots, J, \\
M=2^{J}, & i=m+k+1 .
\end{array}
$$

It is to be noted that $J$ is the maximal resolution level. It is observed that the Haar wavelets and modified Haar wavelets are related as $\mathcal{H}_{i}(x)=x^{c} h_{i}(x)$, where $h_{i}(x)$ is the $i$ th Haar wavelet [32].

To approximate Hadamard-type integral by Haar wavelets, the Hadamard-type integrals of Haar wavelets is required. Using the definition of $\mathcal{H}_{i}(x)$, these integrals can be analytically evaluated using Lemma 2.6.

For $x \in[a, b]$,

$$
\mathfrak{I}_{a, c}^{\alpha} \mathcal{H}_{0}(x)=\frac{1}{\Gamma(\alpha)} \int_{a}^{x}\left(\frac{s}{x}\right)^{c}\left(\log \frac{x}{s}\right)^{\alpha-1} H_{0}(s) \frac{d s}{s}=\frac{x^{-c}}{\Gamma(\alpha+1)}\left(\log \frac{x}{a}\right)^{\alpha} .
$$


For $x \in\left[\eta_{1}(i), \eta_{2}(i)\right)$,

$$
\mathfrak{I}_{a, c}^{\alpha} \mathcal{H}_{i}(x)=\frac{1}{\Gamma(\alpha)} \int_{\eta_{1}}^{x}\left(\frac{s}{x}\right)^{c}\left(\log \frac{x}{s}\right)^{\alpha-1} H_{i}(s) \frac{d s}{s}=\frac{x^{-c}}{\Gamma(\alpha+1)}\left(\log \frac{x}{\eta_{1}}\right)^{\alpha} .
$$

Now, for $x \in\left[\eta_{2}(i), \eta_{3}(i)\right)$,

$$
\begin{aligned}
\mathfrak{I}_{a, c}^{\alpha} \mathcal{H}_{i}(x) & =\frac{x^{-c}}{\Gamma(\alpha)}\left[\int_{\eta_{1}}^{\eta_{2}}\left(\log \frac{x}{s}\right)^{\alpha-1} \frac{d s}{s}-\int_{\eta_{2}}^{x}\left(\log \frac{x}{s}\right)^{\alpha-1} \frac{d s}{s}\right] \\
& =\frac{x^{-c}}{\Gamma(\alpha+1)}\left[\left(\log \frac{x}{\eta_{1}}\right)^{\alpha}-2\left(\log \frac{x}{\eta_{2}}\right)^{\alpha}\right] .
\end{aligned}
$$

Finally, for $x \geq n_{3}(i)$,

$$
\begin{aligned}
\mathfrak{I}_{a, c}^{\alpha} \mathcal{H}_{i}(x) & =\frac{x^{-c}}{\Gamma(\alpha)}\left[\int_{\eta_{1}}^{\eta_{2}}\left(\log \frac{x}{s}\right)^{\alpha-1} \frac{d s}{s}-\int_{\eta_{2}}^{\eta_{3}}\left(\log \frac{x}{s}\right)^{\alpha-1} \frac{d s}{s}\right] \\
& =\frac{x^{-c}}{\Gamma(\alpha+1)}\left[\left(\log \frac{x}{\eta_{1}}\right)^{\alpha}-2\left(\log \frac{x}{\eta_{2}}\right)^{\alpha}+\left(\log \frac{x}{\eta_{3}}\right)^{\alpha}\right] .
\end{aligned}
$$

In summary, we have

$$
\mathfrak{I}_{a, c}^{\alpha} \mathcal{H}_{i}(x)=\frac{x^{-c}}{\Gamma(\alpha+1)} \begin{cases}0, & x<\eta_{1}(i) ; \\ \left(\log \frac{x}{\eta_{1}}\right)^{\alpha}, & x \in\left[\eta_{1}(i), \eta_{2}(i)\right) ; \\ \left(\log \frac{x}{\eta_{1}}\right)^{\alpha}-2\left(\log \frac{x}{\eta_{2}}\right)^{\alpha}, & x \in\left[\eta_{2}(i), \eta_{3}(i)\right) ; \\ \left(\log \frac{x}{\eta_{1}}\right)^{\alpha}-2\left(\log \frac{x}{\eta_{2}}\right)^{\alpha}+\left(\log \frac{x}{\eta_{3}}\right)^{\alpha}, & x \geq \eta_{3} .\end{cases}
$$

A function $f \in L_{1}[a, b]$ is represented by Haar-type wavelet as

$$
f(x)=\sum_{l=0}^{\infty} C_{l} \mathcal{H}_{l}(x)
$$

where

$$
C_{l}=\int_{a}^{b} f(x) \mathcal{H}_{l}(x) d x
$$

In applications, a function $f$ is approximated by the $k$ th partial sum of the Haar series as

$$
f(x)=f_{k}(x)=\sum_{l=0}^{k} C_{l} \mathcal{H}_{l}(x) .
$$

We now introduce an operational matrix of Hadamard-type integration using the modified Haar wavelets. Let $\mathcal{M}(x)=\left[\mathcal{H}_{0}(x), \mathcal{H}_{1}(x), \mathcal{H}_{2}(x), \ldots, \mathcal{H}_{k-1}(x)\right]$, then at collocation points are

$$
\mathcal{M}_{k \times k}=\left[\mathcal{M}_{k}(x)\left(\frac{1}{2 k}\right) \mathcal{M}_{k}(x)\left(\frac{3}{2 k}\right) \cdots \mathcal{M}_{k}(x)\left(\frac{2 k-1}{2 k}\right)\right] .
$$


The Hadamard-type fractional integration of Haar wavelets yields

$$
\mathfrak{I}_{a, c}^{\alpha} \mathcal{M}_{k}(x)=\mathcal{P}_{k \times k} \mathcal{M}_{k}(x)
$$

where $\mathcal{P}_{k \times k}$ is the operational matrix of Hadamard-type integration for Haar type wavelets. The approximation of the fractional Hadamard-type integral is given as follows:

$$
\mathfrak{I}_{a, c}^{\alpha} f(x)=\mathfrak{I}_{a, c}^{\alpha} \sum_{l=0}^{k} C_{l} \mathcal{H}_{l}(x)=\sum_{l=0}^{k} C_{l} \Im_{a, c}^{\alpha} \mathcal{H}_{l}(x)=C \mathcal{P} \mathcal{M}
$$

Example 3.1 Let $f(x)=x^{-c}\left(\log \frac{x}{a}\right)^{\alpha}$. Then we have

$$
\mathfrak{I}_{a, c}^{\alpha} f(x)=x^{-c} \frac{\Gamma(\alpha+1)}{\Gamma(2 \alpha+1)}\left(\log \frac{x}{a}\right)^{2 \alpha}
$$

The numerical and exact values of the integral $\mathfrak{\Im}_{a, f}^{\alpha} f(x)$ are shown in Table 1. Plots for different values of $c$ along with graphs of absolute error are shown in Figs. 1 and 2.

Example 3.2 Let $g(x)=x^{\alpha-c} \sin (3 \alpha x)$. The Hadamard-type fractional integral of $g$ is computed for $1 \leq \alpha \leq 2,1 \leq x \leq 8$. The graph for numerical values is presented in Fig. 3 . The resolution level is taken as $J=6$.

Table 1 Exact and numerical values for $J=5, c=1, \alpha=1.5$

\begin{tabular}{llll}
\hline$x$ & Exact values of $\mathfrak{\Im}_{a, c}^{\alpha} f(x)$ & Numerical values of $\mathfrak{\Im}_{a, c}^{\alpha} f(x)$ & Absolute error \\
\hline 1.1406 & 0.0068 & 0.008137 & $1.337 \times 10^{-3}$ \\
2.2656 & 0.0711 & 0.072241 & $1.141 \times 10^{-3}$ \\
3.1094 & 0.1190 & 0.119924 & $9.24 \times 10^{-4}$ \\
4.2344 & 0.1681 & 0.168898 & $7.98 \times 10^{-4}$ \\
5.0781 & 0.1956 & 0.196315 & $7.15 \times 10^{-4}$ \\
6.2031 & 0.2232 & 0.223817 & $6.17 \times 10^{-4}$ \\
\hline
\end{tabular}

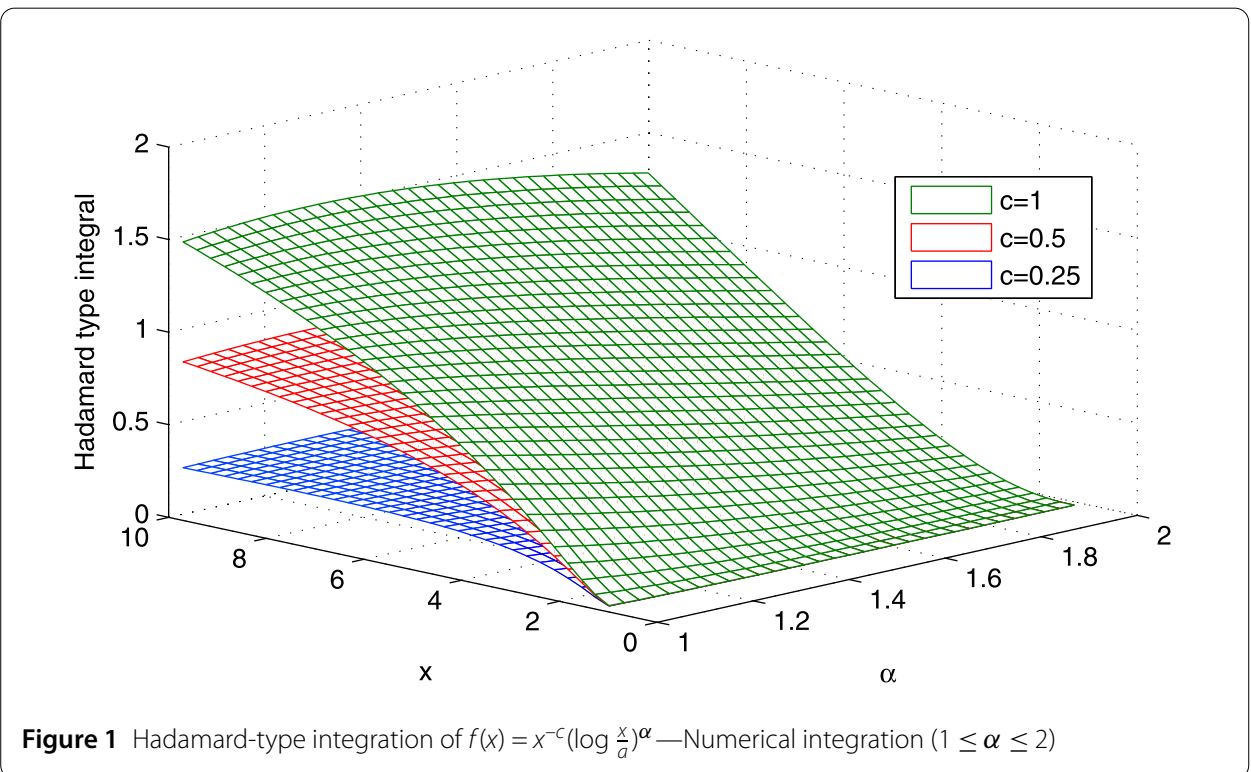



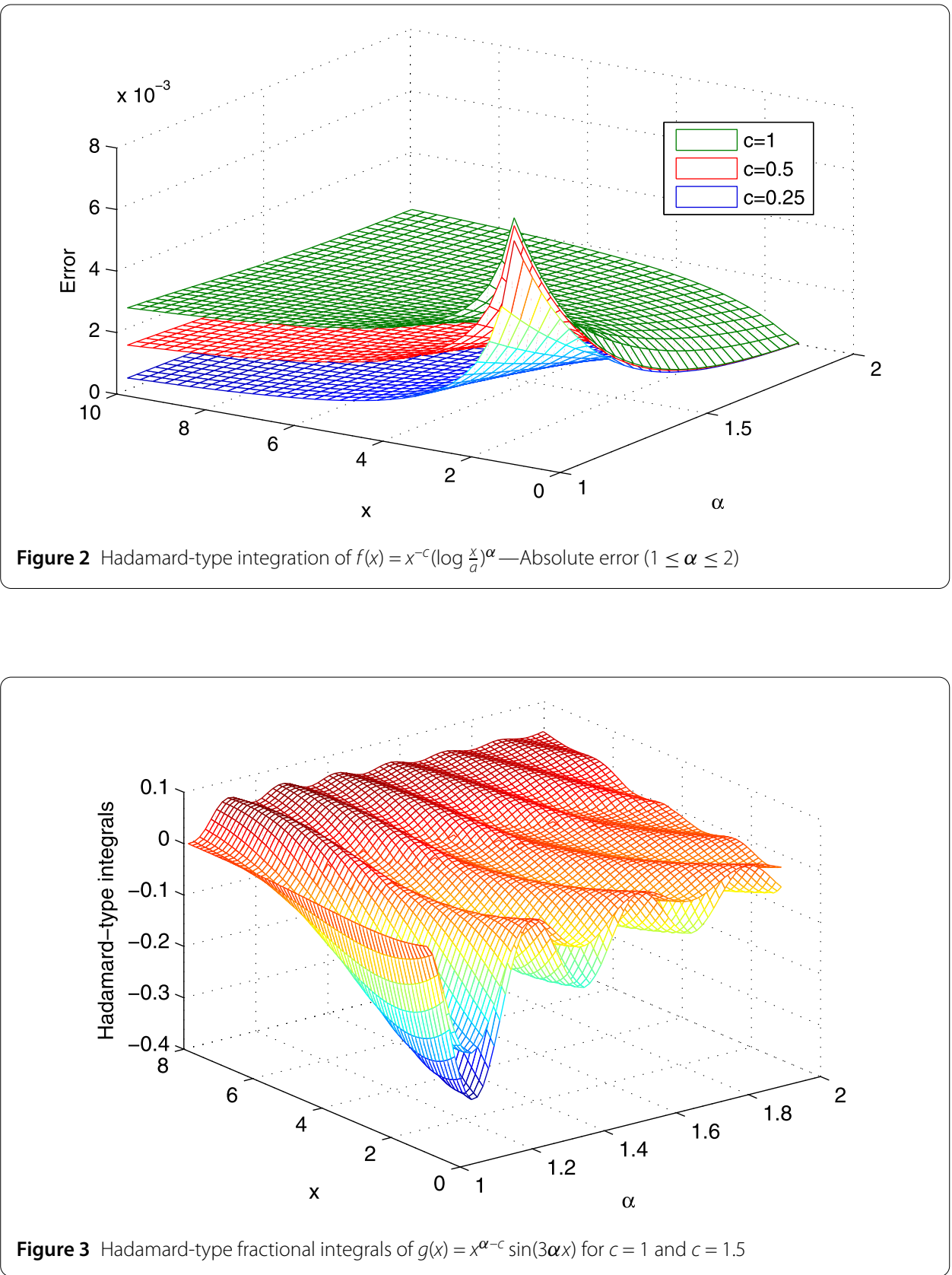

\section{Properties of Hadamard-type fractional operators}

In the following identity, a relationship between Hadamard-type fractional derivative of order $\alpha>0$ and a Caputo modification of Hadamard-type derivative of the same order is established.

To simplify the calculations, a variant of integration by parts formula in notations of Hadamard-type calculus is introduced. For $f, g \in \mathfrak{L}_{c}^{1}[a, b]$, the following relation holds:

$$
\int_{a}^{x}\left(\frac{x}{s}\right)^{c} \mathfrak{D}_{c}\left(s^{-c} f(s)\right) g(s) \frac{d s}{s}=\left.x^{-c} f(s) g(s)\right|_{a} ^{x}-\int_{a}^{x}\left(\frac{x}{s}\right)^{c} f(s) \mathfrak{D}_{c}\left(s^{-c} g(s)\right) \frac{d s}{s} .
$$


Theorem 4.1 Let $f \in \operatorname{AC}_{c}^{m}[a, b]$ and $m-1<\alpha \leq m, m \in \mathbb{N}$. Then

$$
{ }^{C} \mathfrak{D}_{a, c}^{\alpha} f(x)=\frac{1}{\Gamma(m-\alpha)} \int_{a}^{x}\left(\frac{s}{x}\right)^{c}\left(\log \frac{x}{s}\right)^{m-\alpha-1} \mathfrak{D}_{c}^{m} f(s) \frac{d s}{s}=\mathfrak{I}_{a, c}^{m-\alpha} \mathfrak{D}_{c}^{m} f(x) .
$$

Proof The proof follows from Eq. (4.5) and Theorem 2.1 in [22].

For composition properties of Hadamard-type integral and Riemann-Liouville Hadamard-type derivative, the reader is referred to [13-16]. In the following section, we present an important property for Caputo-Hadamard-type operators, which plays an important role in the qualitative analysis of fractional differential equations.

Lemma 4.2 Assume $f \in \operatorname{AC}_{c}^{m}[a, b]$ and $m \in \mathbb{N}$. Then

$$
\mathfrak{I}_{a, c}^{m} \mathfrak{D}_{c}^{m} f(x)=f(x)-a^{c} x^{-c} \sum_{k=0}^{m-1} \frac{\mathfrak{D}_{c}^{k} f(a)}{\Gamma(k+1)}\left(\log \frac{x}{a}\right)^{k} .
$$

Proof For $m=1$, by definitions of $\mathfrak{I}_{a, c}^{1}$ and $\mathfrak{D}_{c}^{1}$, we have

$$
\mathfrak{I}_{a, c}^{1} \mathfrak{D}_{a}^{1} f(x)=x^{-c} \int_{a}^{x}\left(s^{c} f(s)\right)^{\prime} d s=f(x)-a^{c} x^{-c} f(a)
$$

Assume that identity (4.2) holds for $m-1$, where $m>1$. Then, by the semigroup property for $\mathfrak{I}_{a, c}$ and $\mathfrak{D}_{c}$, we have

$$
\mathfrak{I}_{a, c}^{m} \mathfrak{D}_{c}^{m} f(x)=\mathfrak{I}_{a, c}^{1}\left(\mathfrak{I}_{a, c}^{m-1} \mathfrak{D}_{c}^{m-1}\right) \mathfrak{D}_{c}^{1} f(x)
$$

Finally, using (4.2), we get

$$
\begin{aligned}
\mathfrak{I}_{a, c}^{m} \mathfrak{D}_{c}^{m} f(x) & =\mathfrak{I}_{a, c}^{1} \mathfrak{D}_{c}^{1} f(x)-a^{c} x^{-c} \sum_{k=0}^{m-2} \frac{\mathfrak{D}_{c}^{k+1} f(a)}{\Gamma(k+2)}\left(\log \frac{x}{a}\right)^{k+1} \\
& =f(x)-a^{c} x^{-c} f(a)-a^{c} x^{-c} \sum_{k=1}^{m-1} \frac{\mathfrak{D}_{c}^{k} f(a)}{\Gamma(k+1)}\left(\log \frac{x}{a}\right)^{k} \\
& =f(x)-a^{c} x^{-c} \sum_{k=0}^{m-1} \frac{\mathfrak{D}_{c}^{k} f(a)}{\Gamma(k+1)}\left(\log \frac{x}{a}\right)^{k} .
\end{aligned}
$$

It is a common practice to transform an initial value problem or boundary value problem to an equivalent Volterra integral equation to perform qualitative analysis. In this context, the following property for Caputo-Hadamard-type operator, which is very useful for the qualitative analysis of Caputo-Hadamard differential equations, is established.

Theorem 4.3 Assume $f \in \mathrm{AC}_{c}^{m}[a, b]$ and $m-1<\alpha \leq m, m \in \mathbb{N}$. Then

$$
\mathfrak{I}_{a, c}^{\alpha C} \mathfrak{D}_{a, c}^{\alpha} f(x)=f(x)-a^{c} x^{-c} \sum_{k=0}^{m-1} \frac{\mathfrak{D}_{c}^{k} f(a)}{\Gamma(k+1)}\left(\log \frac{x}{a}\right)^{k}
$$


Proof The proof follows from the definition of ${ }^{C} \mathfrak{D}_{c}^{\alpha}$, the semigroup property of $\mathfrak{I}_{a, c}^{\alpha}$, and Lemma 4.

For convenience, the Leibniz rule for Hadamard-type calculus is stated. The proof of the following lemma follows from the standard Leibniz rule, where the notation

$$
\mathfrak{D}_{c, x} f(x, t)=x^{-c}\left(x \frac{\partial}{\partial x}\right) x^{c} f(x, t)
$$

is used to represent the partial derivative.

Lemma 4.4 Assume that $h(x, t)$ and $\frac{\partial}{\partial x} h(x, t) \in\left(\mathrm{AC}_{c}^{m}[a, b]\right)^{2}$. Then

$$
\mathfrak{D}_{c} \int_{a}^{x}\left(\frac{s}{x}\right)^{c} h(x, s) \frac{d s}{s}=h(x, x)-\int_{a}^{x}\left(\frac{s}{x}\right)^{c} \mathfrak{D}_{c, x} h(x, s) \frac{d s}{s} .
$$

Lemma 4.5 If $\mu \geq \alpha$ and $f \in \operatorname{AC}_{c}^{m}[a, b]$, then ${ }^{C} \mathfrak{D}_{c}^{\alpha} \mathfrak{I}_{a, c}^{\mu} f(x)=\mathfrak{I}_{a, c}^{\mu-\alpha} f(x)$.

Proof By Definition 2.8, Lemma 4.4, and the semigroup property of $\mathfrak{I}_{c}^{\alpha}$, we have

$$
{ }^{C} \mathfrak{D}_{c}^{\alpha} \mathfrak{I}_{a, c}^{\mu} f(x)=\mathfrak{I}_{c}^{m-\alpha} \mathfrak{D}_{c}^{m} \mathfrak{I}_{a, c}^{\mu} f(x)=\mathfrak{I}_{c}^{m-\alpha} \mathfrak{I}_{a, c}^{m-\mu} f(x)=\mathfrak{I}_{a, c}^{\mu-\alpha} f(x)
$$

Lemma 4.6 Let $f \in \operatorname{AC}_{c}^{m}[a, b]$ and $\alpha \geq 0, \beta \geq 0$ such that $m-1<\alpha \leq m$ and $n-1<\beta \leq n$. Then

$$
{ }^{C} \mathfrak{D}_{a, c}^{\alpha}\left({ }^{C} \mathfrak{D}_{a, c}^{\beta} f\right)(x)={ }^{C} \mathfrak{D}_{a, c}^{\alpha+\beta} f(x) .
$$

Proof Without loss of generality, let $n \geq m$. Thus $n=m+k, k \in\{0,1,2, \ldots\}$. Since $\alpha+\beta=$ $m+n$, then by the definition of $\mathfrak{D}_{a, c}^{\alpha}$ and the semigroup property of fractional integral $\mathfrak{I}_{a}^{\alpha}$, we have

$$
\begin{aligned}
{ }^{C} \mathfrak{D}_{a, c}^{\alpha}\left({ }^{C} \mathfrak{D}_{a, c}^{\beta} f\right)(x) & =\mathfrak{I}_{a, c}^{m-\alpha} \mathfrak{D}_{c}^{m}\left({ }^{C} \mathfrak{D}_{a, c}^{\beta} f(x)\right) \\
& =\mathfrak{I}_{a, c}^{m-\alpha} \mathfrak{D}_{c}^{m}\left(\mathfrak{I}_{a, c}^{n-\beta} \mathfrak{D}_{c}^{n} f(x)\right) \\
& =\mathfrak{I}_{a, c}^{m-\alpha} \mathfrak{D}_{c}^{m}\left(\mathfrak{I}_{a, c}^{m+k-\beta} \mathfrak{D}_{c}^{m+k} f(x)\right) \\
& =\mathfrak{I}_{a, c}^{m-\alpha} \mathfrak{D}_{c}^{m} \mathfrak{I}_{a, c}^{m-\beta} \mathfrak{I}_{a, c}^{k} \mathfrak{D}_{c}^{m+k} f(x) \\
& =\mathfrak{I}_{a, c}^{m-\alpha} \mathfrak{D}_{a, c}^{\beta} \mathfrak{I}_{a, c}^{k} \mathfrak{D}_{c}^{m+k} f(x) \\
& =\mathfrak{I}_{a, c}^{m-\alpha-\beta} \mathfrak{I}_{a, c}^{\beta} \mathfrak{D}_{a, c}^{\beta} \mathfrak{I}_{a, c}^{k} \mathfrak{D}_{c}^{m+k} f(x) .
\end{aligned}
$$

By Theorem 4.3, we have

$$
\begin{aligned}
{ }^{c} \mathfrak{D}_{a, c}^{\alpha}\left({ }^{C} \mathfrak{D}_{a, c}^{\beta} f\right)(x)= & \mathfrak{I}_{a, c}^{m-\alpha-\beta}\left[\mathfrak{I}_{a, c}^{k} \mathfrak{D}_{c}^{m+k} f(x)\right. \\
& \left.-a^{c} x^{-c} \sum_{j=0}^{m-1} \frac{\mathfrak{D}_{c}^{m-j-1} \mathfrak{I}_{a, c}^{m-\beta} \mathfrak{I}_{a, c}^{k} \mathfrak{D}_{c}^{m+k} f(a)}{\Gamma(\beta-j)}\left(\log \frac{x}{a}\right)^{\beta-j-1}\right] .
\end{aligned}
$$


Let $\lceil\beta\rceil=n=m+k$ and since

$$
\mathfrak{I}_{a, c}^{m-\beta} \mathfrak{I}_{a, c}^{k} \mathfrak{D}_{c}^{m+k} f(a)=\mathfrak{I}_{a, c}^{\lceil\beta\rceil-\beta} \mathfrak{D}_{c}^{\lceil\beta\rceil} f(a)={ }^{C} \mathfrak{D}_{a, c}^{\beta} f(a) .
$$

Therefore

$$
{ }^{C} \mathfrak{D}_{a, c}^{\alpha}\left({ }^{C} \mathfrak{D}_{a, c}^{\beta} f\right)(x)=\mathfrak{I}_{a, c}^{m-(\alpha \beta)}\left[\mathfrak{I}_{a, c}^{k} \mathfrak{D}_{c}^{m+k} f(x)-a^{c} x^{-c} \sum_{j=0}^{m-1} \frac{\mathfrak{D}_{c}^{m-j-1} C \mathfrak{D}_{a, c}^{\beta} f(a)}{\Gamma(\beta-j)}\left(\log \frac{x}{a}\right)^{\beta-j-1}\right] .
$$

For $\beta \geq 0$, we have ${ }^{C} \mathfrak{D}_{a, c}^{\beta} f(a)=0$. Thus

$$
\begin{aligned}
{ }^{C} \mathfrak{D}_{a, c}^{\alpha}\left({ }^{C} \mathfrak{D}_{a, c}^{\beta} f\right)(x) & =\mathfrak{I}_{a, c}^{m-(\alpha+\beta)}\left(\mathfrak{I}_{a, c}^{k} \mathfrak{D}_{c}^{m+k} f(x)\right) \\
& =\mathfrak{I}_{a, c}^{m+k-(\alpha+\beta)} \mathfrak{D}_{c}^{m+k} f(x) \\
& ={ }^{C} \mathfrak{D}_{a, c}^{\alpha+\beta} f(x),
\end{aligned}
$$

which establishes the required identity.

Remark 4.7 From Definition 2.4, we notice an obvious relation

$$
\mathfrak{I}_{a, c}^{\alpha} f(x)=x^{-c} \mathfrak{I}_{a}^{\alpha}\left(x^{c} f(x)\right)
$$

between Hadamard-type integral $\mathfrak{I}_{a, c}^{\alpha}$ and Hadamard integral $\mathfrak{I}_{a}^{\alpha}$. Also, from Definition 2.7 and Eq. (4.3), Hadamard-type derivative $\mathfrak{D}_{a, c}^{\alpha}$ and Hadamard derivative $\mathfrak{D}_{c}^{\alpha}$ are related as follows:

$$
\mathfrak{D}_{a, c}^{\alpha} f(x)=x^{-c} \mathfrak{D}_{a}^{\alpha}\left(x^{c} f(x)\right) .
$$

A similar relation holds between a Caputo modification of Hadamard-type derivative ${ }^{C} \mathfrak{D}_{a, c}^{\alpha}$ and a Caputo modification of Hadamard derivative ${ }^{C} \mathfrak{D}_{a}^{\alpha}$, that is,

$$
{ }^{C} \mathfrak{D}_{a, c}^{\alpha} f(x)=x^{-c C} \mathfrak{D}_{a}^{\alpha}\left(x^{c} f(x)\right) .
$$

\section{Taylor's formula}

In this section, Taylor's formula involving Caputo-Hadamard-type derivative is discussed. To establish the main theorem, the following result is required.

Theorem 5.1 Let $0<\alpha \leq 1$ and $f \in \mathrm{AC}[a, b]$ such that ${ }^{C} \mathfrak{D}_{a, f}^{\alpha} f \in C[a, b]$. Then, for all $x \in$ $[a, b]$, there exists $\xi \in[a, b]$ such that, for $\xi \in[a, x]$,

$$
f(x)=a^{c} x^{-c} f(a)+\frac{1}{\Gamma(\alpha+1)}\left(\log \frac{x}{a}\right)^{\alpha}\left(\frac{\xi}{x}\right)^{c} C \mathfrak{D}_{a, \delta}^{\alpha} f(\xi) .
$$

Proof Since $0<\alpha \leq 1$, we have by Theorem 4.3

$$
\mathfrak{I}_{a, c}^{\alpha}\left({ }^{C} \mathfrak{D}_{a, c}^{\alpha} f\right)(x)=f(x)-a^{c} x^{-c} f(a) .
$$


Using the definition of $\mathfrak{I}_{a, c}^{\alpha}$ and by the integral mean value theorem, we obtain

$$
\begin{aligned}
\mathfrak{I}_{a, c}^{\alpha}\left({ }^{C} \mathfrak{D}_{a, c}^{\alpha} f\right)(x) & =\frac{1}{\Gamma(\alpha)} \int_{a}^{x}\left(\frac{t}{x}\right)^{c}\left(\log \frac{x}{t}\right)^{\alpha-1} C \mathfrak{D}_{a, c}^{\alpha} f(t) \frac{d t}{t} \\
& =\frac{1}{\Gamma(\alpha+1)}\left(\frac{\xi}{x}\right)^{c}{ }^{C} \mathfrak{D}_{a, c}^{\alpha} f(\xi)\left(\log \frac{x}{a}\right)^{\alpha} \\
& =\frac{1}{\Gamma(\alpha+1)}\left(\frac{\xi}{x}\right)^{c}\left(\log \frac{x}{a}\right)^{\alpha}{ }^{C} \mathfrak{D}_{a, c}^{\alpha} f(\xi),
\end{aligned}
$$

where $\xi=(a, x)$. Hence, we get

$$
f(x)=a^{c} x^{-c} f(a)+\frac{1}{\Gamma(\alpha+1)}\left(\frac{\xi}{x}\right)^{c}\left(\log \frac{x}{a}\right)^{\alpha}{ }^{C} \mathfrak{D}_{a, f}^{\alpha} f(\xi)
$$

Proposition 5.2 If $0<\alpha \leq 1, j \in \mathbb{N}$, and $f \in \mathrm{AC}_{c}^{m}[a, b]$, then

$$
\mathfrak{I}_{a, c}^{j \alpha C} \mathfrak{D}_{a, c}^{j \alpha} f(x)-\mathfrak{I}_{a, c}^{(j+1) \alpha} C_{\mathfrak{D}_{a, c}^{(j+1) \alpha}} f(x)=\frac{a^{c} x^{-c}}{\Gamma(j \alpha+1)}\left(\log \frac{x}{a}\right)^{j \alpha} C \mathfrak{D}_{a, c}^{j \alpha} f(a) .
$$

Proof By Lemma 4.6, Theorem 4.3, and Eq. (5.1), we have

$$
\begin{aligned}
\mathfrak{I}_{a, c}^{j \alpha C} \mathfrak{D}_{a, c}^{j \alpha} f(x)-\mathfrak{I}_{a, c}^{(j+1) \alpha C} \mathfrak{D}_{a, c}^{(j+1) \alpha} f(x) & =\mathfrak{I}_{a, c}^{j \alpha}\left[{ }^{C} \mathfrak{D}_{a, c}^{j \alpha} f(x)-\mathfrak{I}_{a, c}^{\alpha}{ }^{C} \mathfrak{D}_{a, c}^{(j+1) \alpha} f(x)\right] \\
& =\mathfrak{I}_{a, c}^{j \alpha}\left[{ }^{C} \mathfrak{D}_{a, c}^{j \alpha} f(x)-\mathfrak{I}_{a, c}^{\alpha}{ }^{C} \mathfrak{D}_{a, c}^{\alpha}\left({ }^{C} \mathfrak{D}_{a, c}^{j \alpha} f(x)\right)\right] \\
& =a^{c} \mathfrak{I}_{a, c}^{j \alpha}\left(x^{-c C} \mathfrak{D}_{a, c}^{j \alpha} f(a)\right) \\
& =\frac{a^{c} x^{-c}}{\Gamma(j \alpha+1)}\left(\log \frac{x}{a}\right)^{j \alpha}{ }^{C} \mathfrak{D}_{a, c}^{j \alpha} f(a) .
\end{aligned}
$$

Theorem 5.3 Let $0<a<b<\infty$ and $0<\alpha \leq 1$. Let $m$ be an arbitrary nonnegative integer. Let $f \in \operatorname{AC}_{c}^{m}[a, b]$ and suppose ${ }^{C} \mathfrak{D}_{a, c}^{(j+1) \alpha} f \in C[a, b]$. Then, for $\xi=[a, x]$, Taylor's formula involving Caputo-Hadamard-type fractional derivatives writes

$$
f(x)=\sum_{j=0}^{m} \frac{a^{c} x^{-c}}{\Gamma(j \alpha+1)}\left(\log \frac{x}{a}\right)^{j \alpha}{ }^{C} \mathfrak{D}_{a, f}^{j \alpha} f(a)+\frac{{ }^{C} \mathfrak{D}_{a, c}^{(m+1) \alpha} f(\xi)}{\Gamma((m+1) \alpha+1)}\left(\frac{\xi}{x}\right)^{c}\left(\log \frac{x}{a}\right)^{(m+1) \alpha}
$$

Proof Using Proposition 5.2, we have

$$
\begin{aligned}
\sum_{j=0}^{m} \frac{a^{c} x^{-c}}{\Gamma(j \alpha+1)}\left(\log \frac{x}{a}\right)^{j \alpha} C_{\mathfrak{D}_{a, c}^{j \alpha}} f(a) & =\sum_{j=0}^{m}\left(\mathfrak{J}_{a, c}^{j \alpha} C_{\mathfrak{D}_{a, c}}^{j \alpha} f(x)-\mathfrak{I}_{a, c}^{(j+1) \alpha C} C_{\mathfrak{D}_{a, c}^{(j+1) \alpha}} f(x)\right) \\
& =\mathfrak{I}_{a, c}^{0} C_{\mathfrak{D}_{a, c}^{0}} f(x)-\mathfrak{I}_{a, c}^{(m+1) \alpha C} \mathfrak{D}_{a, c}^{(m+1) \alpha} f(x) .
\end{aligned}
$$

It follows

$$
f(x)=\sum_{j=0}^{m} \frac{a^{c} x^{-c}}{\Gamma(j \alpha+1)}\left(\log \frac{x}{a}\right)^{j \alpha} C_{\mathfrak{D}_{a, c}^{j \alpha}} f(a)+\mathfrak{I}_{a, c}^{(m+1) \alpha} C_{\mathfrak{D}_{a, c}^{(m+1) \alpha}} f(x)
$$


Replacing $\alpha$ by $(m+1) \alpha$ in Eq. (5.2), we get

$$
\mathfrak{I}_{a, c}^{(m+1) \alpha} C_{\mathfrak{D}_{a, c}^{(m+1) \alpha}} f(x)=\frac{1}{\Gamma((m+1) \alpha+1)}\left(\frac{\xi}{x}\right)^{c}\left(\log \frac{x}{a}\right)^{(m+1) \alpha} C_{\mathfrak{D}_{a, c}^{(m+1) \alpha}} f(\xi) .
$$

Consequently, (5.3) follows by substituting (5.5) in (5.4).

Example 5.4 For $0<\alpha \leq 1$, let $a, \mu$ be nonzero real numbers and $f \in C[a, \infty)$. Consider the Caputo-Hadamard-type fractional differential equation

$$
{ }^{C} \mathfrak{D}_{a}^{\alpha} f(x)-\mu f(x)=0 .
$$

Assume that $f$ has the power series representation

$$
f(x)=\sum_{j=0}^{\infty} q_{j} x^{-c}\left(\log \frac{x}{a}\right)^{j \alpha}
$$

Applying ${ }^{C} \mathfrak{D}_{a}^{\alpha}$ on both sides of (5.7) and using Lemma 2.9, we have

$$
\begin{aligned}
{ }^{C} \mathfrak{D}_{a}^{\alpha} f(x) & =\sum_{j=0}^{\infty} q_{j}{ }^{C} \mathfrak{D}_{a}^{\alpha} x^{-c}\left(\log \frac{x}{a}\right)^{j \alpha} \\
& =\sum_{j=1}^{\infty} q_{j} \frac{\Gamma(j \alpha+1)}{\Gamma((j-1) \alpha+1)} x^{-c}\left(\log \frac{x}{a}\right)^{j \alpha} \\
& =\sum_{j=0}^{\infty} q_{j+1} \frac{\Gamma((j+1) \alpha+1)}{\Gamma(j \alpha+1)} x^{-c}\left(\log \frac{x}{a}\right)^{j \alpha} .
\end{aligned}
$$

Using Eqs. (5.7) and (5.8) in (5.6), we have

$$
q_{j+1}=\frac{\Gamma(j \alpha+1)}{\Gamma((j+1) \alpha+1)} \mu q_{j}, \quad j=0,1,2, \ldots
$$

Solving the difference equation (5.9) for $q_{j}$, we get

$$
q_{j}=\mu^{j} \frac{1}{\Gamma(j \alpha+1)} q_{0}
$$

Substituting $q_{j}$ from Eq. (5.10) into Eq. (5.7), we have

$$
f(x)=q_{0} x^{-c} \sum_{j=0}^{\infty} \frac{\mu^{j}}{\Gamma(j \alpha+1)}\left(\log \frac{x}{a}\right)^{j \alpha}=q_{0} x^{-c} E_{\alpha}\left(\mu\left(\log \frac{x}{a}\right)^{\alpha}\right),
$$

where $E_{\alpha}(\cdot)$ is the Mittag-Leffler function. 
Availability of data and materials

Not applicable.

Competing interests

The authors declare that they have no competing interests.

\section{Authors' contributions}

All the authors contributed in the investigation. MR proposed the study. MR and MS finalized the manuscript. All the authors read and approved the submission of the article in this form.

\section{Publisher's Note}

Springer Nature remains neutral with regard to jurisdictional claims in published maps and institutional affiliations.

Received: 21 September 2019 Accepted: 22 April 2020 Published online: 15 May 2020

\section{References}

1. Katugampola, U.N.: A new approach to generalized fractional derivatives. Bull. Math. Anal. Appl. 6(4), 1-15 (2014)

2. Liouville, J.: Mémoire sur quelques Quéstions de Géometrie et de Mécanique, et sur un nouveau genre de Calcul pour résoudre ces Quéstions. J. Éc. Polytech. XIII, XXle cahier, 1-69 (1832)

3. Samko, S.G., Kilbas, A.A., Marichev, O.I.: Fractional Integrals and Derivatives: Theory and Applications. Gordon \& Breach, Amsterdam (1993)

4. Munkhamar, M.: Riemann-Liouville fractional derivatives and the Taylor-Riemann series. Uppsala University, Department of Mathematics

5. Oldham, K.B., Spanier, J.: The Fractional Calculus. Academic Press, New York (1974)

6. Podlubny, I.: Fractional Differential Equations. Academic Press, New York (1999)

7. Kilbas, A.A., Srivastava, H.M., Trujillo, J.J.: Theory and Applications of Fractional Differential Equations. Elsevier, Amsterdam (2006)

8. Atangana, A., Botha, J.F.: Generalized groundwater flow equation using the concept of variable order derivative. Bound. Value Probl. 2013, 53 (2013). https://doi.org/10.1186/1687-2770-2013-53

9. Miller, K.S., Ross, B.: An Introduction to the Fractional Calculus and Fractional Differential Equations. Wiley, New York (1993)

10. Zaslavsky, G.M.: Hamiltonian Chaos and Fractional Dynamics. Oxford University Press, London (2005)

11. Atangana, A., Secer, A.: The time-fractional coupled-Korteweg-de-Vries equations. Abstr. Appl. Anal. 2013, Article ID $947986(2013)$

12. Hadamard, J.: Essai sur l'etude des fonctions donnees par leur developpment de Taylor. J. Math. Pures Appl. 4(8), 101-186 (1892)

13. Kilbas, A.: Hadamard-type fractional calculus. J. Korean Math. Soc. 38(6), 1191-1204 (2001)

14. Butzer, P.L., Kilbas, A.A., Trujillo, J.J.: Compositions of Hadamard-type fractional integration operators and the semigroup property. J. Math. Anal. Appl. 269, 387-400 (2002)

15. Butzer, P.L., Kilbas, A.A., Trujillo, J.J.: Fractional calculus in the Mellin setting and Hadamard-type fractional integrals. J. Math. Anal. Appl. 269, 1-27 (2002)

16. Butzer, P.L., Kilbas, A.A., Trujillo, J.J.: Mellin transform analysis and integration by parts for Hadamard-type fractional integrals. J. Math. Anal. Appl. 270, 1-15 (2002)

17. Caputo, M.: Linear model of dissipation whose $Q$ is almost frequency independent. II. Geophys. J. Int. 13(5), 529-539 (1967)

18. Ma, L., Li, C.: On Hadamard fractional calculus. Fractals 25(3), 1750033 (2017). https://doi.org/10.1142/S0218348X17500335

19. Li, C., Qian, D., Chen, Y.: On Riemann-Liouville and Caputo derivatives. Discrete Dyn. Nat. Soc. 2011, Article ID 562494 (2011). https://doi.org/10.1155/2011/562494

20. Almeida, R., Torres, D.F.M.: Calculus of variations with fractional derivatives and fractional integrals. Appl. Math. Lett. 22(12), 1816-1820 (2009)

21. Alsaedi, A., Ahmad, B., Alghanmi, M.: Extremal solutions for generalized Caputo fractional differential equations with Steiltjes-type fractional integro-initial conditions. Appl. Math. Lett. 91, 113-120 (2019)

22. Jarad, F., Abdeljawad, T., Baleanu, D.: Caputo-type modification of the Hadamard fractional derivatives. Adv. Differ Equ. 2012, 142 (2012)

23. Gambo, Y.Y., Jarad, F., Baleanu, D., Abdeljawad, T:: On Caputo modification of the Hadamard fractional derivatives. Adv. Differ. Equ. (2014). https://doi.org/10.1186/1687-1847-2014-10

24. Trujillo, J.J., Rivero, M., Bonilla, B.: On a Riemann-Liouville generalized Taylor's formula. J. Math. Anal. Appl. 231, 255-265 (1999)

25. Odibat, Z.M., Shawagfeh, N.T.: Generalized Taylor's formula. Appl. Math. Comput. 186, 286-293 (2007)

26. Gulsu, M., Ozturk, Y., Anapali, A.: Numerical approach for solving fractional relaxation-oscillation equation. Appl. Math Model. 37, 5927-5937 (2013)

27. Fernandez, A., Baleanu, D.: The mean value theorem and Taylor's theorem for fractional derivatives with Mittag-Leffler kernel. Adv. Differ. Equ. 2018, 86 (2018). https://doi.org/10.1186/s13662-018-1543-9

28. Benjemaa, M.: Taylor's formula involving generalized fractional derivatives. Appl. Math. Comput. 335, 182-195 (2018)

29. Hashish, H., Behiry, S.H., El-Shamy, N.A.: Numerical integration using wavelets. Appl. Math. Comput. 211, 480-487 (2009)

30. Aziz, I., Islam, S., Khan, W.: Quadrature rules for numerical integration based on Haar wavelets and hybrid functions. Comput. Math. Appl. 61, 2770-2781 (2011)

31. Islam, S., Aziz, I., Haq, F.: A comparative study of numerical integration based on Haar wavelets and hybrid functions. Comput. Math. Appl. 59, 2026-2036 (2010)

32. Lepik, U.: Solving integral and differential equations by the aid of non-uniform Haar wavelets. Appl. Math. Comput. 198(1), 326-332 (2008) 